\title{
ÜBER EINEN ZUSAMMENHANG ZWISCHEN DER KONVERGENZ VON POLYNOMFOLGEN UND DER VERTEILUNG IHRER WURZELN.
}

\author{
Von Egon Lindwart (Riga) und Georg Pólya (Budapest).
}

Adunanza del 10 agosto 1913.

Die Untersuchungen, die der eine von uns über diesen Gegenstand vor kurzer Zeit begonnen hat ${ }^{1}$ ), sollen hier zu einem gewissen Abschluss geführt werden. Der im Mittelpunkt stehende Satz I. wurde zuerst von Lindwart formuliert und bewiesen; der einfache Beweis im Text ist von dem erstgefundenen völlig verschieden und rührt von Pólya her.

Satz I. - Es sei eine Folge von Polynomen vorgelegt

$$
\left\{\begin{array}{c}
\Phi_{1}(x), \Phi_{2}(x), \ldots, \Phi_{n}(x), \ldots \\
\Phi_{n}(x)=\left(\mathrm{I}-\frac{x}{\alpha_{n 1}}\right)\left(\mathrm{I}-\frac{x}{\alpha_{n 2}}\right) \ldots\left(\mathrm{I}-\frac{x}{\alpha_{n n}}\right) \\
=\mathrm{I}-a_{n 1} x+a_{n 2} x^{2}-\cdots+(-\mathrm{I})^{n} a_{n n} x^{n}
\end{array}\right.
$$

die in einem Kreis um den Punkt $x=0$ gleichmässig konvergiert. Die Wurzeln von $\Phi_{n}(x)$ seien für alle $n$ der Ungleichung

$$
\sum_{p=1}^{n} \frac{1}{\left|\alpha_{n p}\right|^{k}} \leq M
$$

unterworfen; $k$ und $M$ bedeuten feste positive Zablen.

Dann konvergiert die Polynomfolge notwendigerweise in der ganzen Ebene, und zwar in jedem endlichen Bereiche gleichmässig.

Die Grenzfunktion ist eine ganze Funktion, von endlichem Gescblecht $\leqslant[k]$. Ist $k$ ganz, so ist die Grenzfunktion gleich dem Produkt einer Funktion vom Gescblecht $\leq k-\mathrm{I}$ und der Funktion $e^{\Upsilon^{k}}$ wo $\gamma$ eine Konstante bedeutet.

Beweis: $\lim _{n=\infty} \Phi_{n}(x)=F(x)$ ist regulär analytisch im Innern des besagten Kreises,

I) G. Pósya : a) Über Annäherung durch Polynome mit lauter reellen Wurzeln [Rendiconti del Circolo Matematico di Palermo, Bd. XXXVI (2. Semester 1913), S. 279-295]; b) Über Annäberung durch Polynome, deren sümtliche Wurzeln in einen Winkelraum fallen [Nachrichten von der Kgl. Gesellschaft der Wissenschaften zu Göttingen, Mathematisch-physikalische Klasse, Jahrgang I913, S. 326-330]. 
nach einem Satz von WeIERSTRass. Daher kann

$$
F(x)=\mathrm{I}-a_{1} x+a_{2} x^{2}-a_{3} x^{3}+\cdots
$$

gesetzt werden, und es besteht die Beziehung

$$
\lim _{n=\infty} a_{n p}=a_{p} \text {. }
$$

Ich werde noch die Potenzsummen der reziproken Wurzeln von $\Phi_{n}(x)$ betrachten

$$
s_{n p}=\frac{\mathrm{I}}{\alpha_{n 1}^{p}}+\frac{\mathrm{I}}{\alpha_{n 2}^{p}}+\cdots+\frac{\mathrm{I}}{\alpha_{n n}^{p}} \quad(p=\mathrm{I}, 2,3, \ldots)
$$

$s_{n p}$ ist ein ganzer rationaler Ausdruck in $a_{n 1}, a_{n 2}, \ldots, a_{n p}$; daher ist für alle $n$

(3)

Ich setze

$$
\left|s_{n p}\right|<S_{p} \text {. }
$$

$$
b=\left\{\begin{array}{lll}
{[k]} & \text { wenn } & {[k]<k} \\
k-\mathrm{I}, & \text { wenn } & {[k]=k}
\end{array}\right.
$$

Ich will die bekannte ${ }^{2}$ ), für jedes $z$ bestehende Ungleichung benutzen

$$
|I-z| \leq e^{-r\left(\frac{z}{1}+\frac{z^{2}}{2}+\cdots+\frac{z^{h}}{h}\right)+c|z|^{k}}
$$

$c$ bedeutet eine von $z$ unabhängige Konstante.

Es folgt aus (I) und (4)

$$
\begin{aligned}
& \left|\Phi_{n}(x)\right| \leq \prod_{p=1}^{n} e^{-x\left(\frac{x}{1 \alpha_{n p}}+\frac{x^{2}}{2 \alpha_{n p}^{2}}+\cdots+\frac{x^{b}}{b \alpha_{n p}^{h}}\right)+i\left|\frac{x}{\alpha_{n p}}\right|^{k}} \\
& =e^{-\Re\left(\frac{x s_{n 1}}{L}+\frac{x^{2} s_{n 2}}{2}+\cdots+\frac{x^{h} s_{n h}}{b}\right)+c|x|^{k}} p \stackrel{n}{=} \frac{}{\left|\alpha_{n p}\right|^{k}}
\end{aligned}
$$

(2) und (3) hinzugezogen, gewinnt man die Ungleichung

$$
\left|\Phi_{n}(x)\right| \leq e^{\frac{|x| S_{I}}{5}+\frac{|x|^{2} S_{2}}{2}+\cdots+\frac{|x|^{h} S_{h}}{b}+c M|x|^{k}}
$$

Die Folge

$$
\Phi_{1}(x), \quad \Phi_{2}(x), \ldots, \Phi_{n}(x), \ldots
$$

ist also gleichmässig beschränkt in jedem endlichen Gebiet, und konvergiert gleichmässig in einem Kreis um den Punkt $x=0$. Daraus folgt, gemäss einem grundlegenden Satze von Stieltjes ${ }^{3}$ ), dass sie in der ganzen Ebene konvergiert.

Aus ( 5 ) folgt weiter

$$
|F(x)| \leq e^{\frac{|x| S_{1}}{\mathrm{l}}+\frac{|x|^{2} S_{2}}{2}+\cdots+\frac{|x|^{h} S_{h}}{b}+c M|x|^{k}}
$$

$\left(s^{\prime}\right)$ besagt, nach der Hadamardschen Theorie der ganzen Funktionen, dass $F(x)$ höchstens vom Geschlechte $[k]$ ist.

2) Vgl. z. B. G. Vivanti, Theorie der eindeutigen analytischen Funktionen (deutsch von A. GutzmeR) (Leipzig, Teubner, 1906), S. 233-236.

3) Correspondance d'Hermite et de Stieltjes (Paris, Gauthier-Villars), Bd. II (I905), S. 369-37o. 
Es folgt endlich aus (2) a fortiori

$$
M \supseteq \frac{I}{\left|\alpha_{n 1}\right|^{k}}+\frac{I}{\left|\alpha_{n 2}\right|^{k}}+\cdots+\frac{I}{\left|\alpha_{n p}\right|^{k}} \quad(n=p, p+I, p+2, \ldots) .
$$

Nach einem Satze von Hurwitz muss diese Beziehung auch für irgendwelche $p$ Wurzeln der Grenzfunktion $F(x)$ bestehen, und das heisst, dass die Summe der $k$-ten Potenzen der absolut genommenen reziproken Wurzeln von $F(x)$ konvergiert, und $\leq M$ ist. Damit ist Satz' I. vollständig bewiesen.

Wie aus der Ableitung klar hervorgeht, können die Voraussetzungen des Satzes I. verschiedentlich erweitert und modifiziert werden. Man kann ihn zuerst anstatt für Polynome, für ganze Funktionen vom Geschlechte $<k$ aussprechen, die im Punkte $x=\mathrm{o}$ den Wert $\mathrm{I}$ annehmen und bei welchen die Summe der $k^{\text {ten }}$ Potenzen der absolut genommenen reziproken Wurzeln der gleichen Bedingung (2) der Beschränktheit unterliegt. - Anstatt gleichmässige Konvergenz in dem Kreise um dem Punkt $x=0$, genügt es Konvergenz in irgend welchen unendlich vielen Punkten vorauszusetzen, die einen Häufungspunkt in Endlichen haben, wenn zum Ersatz die weitere Bedingung hinzugefügt wird, dass die ersten $b$ Koeffizienten der $n^{\text {ten }}$ annähernden Funktion, $a_{n \mathrm{I}}$, $a_{n 2}, \ldots, a_{n h}$ für alle $n$ beschränkt bleiben. [Bedingung (2) muss natürlich beibehalten werden]. Bei diesen Voraussetzungen wird an Stelle des Stieltjesschen der weitergehende VITalische Satz ${ }^{4}$ ) benutzt.

Wir wollen aber $z u$ den Anwendungen des Satzes I. schreiten. Man betrachte eine Polynomfolge $\left.\Phi_{1}(x), \Phi_{2}(x), \ldots, \Phi_{n}(x), \ldots{ }^{5}\right)$. Die Wurzeln von $\Phi_{n}(x)$ sollen

$$
\alpha_{n 1}=p_{n 1} e^{i \Im_{n 1}}, \quad \alpha_{n 2}=p_{n 2} e^{i \Im_{n 2}}, \ldots, \alpha_{n n}=p_{n n} e^{i \Im_{n n}}
$$

heissen. (Ist der absolute Betrag $\rho_{n p}$ der Wurzel $x_{n p}$ zufälligerweise $=0$, so soll auch $\operatorname{der}$ Arcus $=0$ gesetzt werden). Um uns im Folgenden kürzer ausdrücken zu können, werden wir die Menge

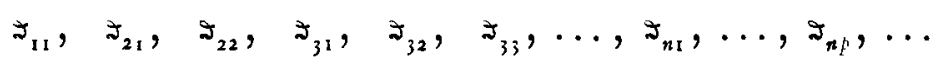

kurz die Arcusmenge der Folge $\Phi_{1}(x), \ldots, \Phi_{n}(x), \ldots$ nennen. Die Arcusmenge wird mod. $2 \pi$ betrachtet, und ist eine Punktmenge im Intervalle $(0,2 \pi)$.

SATz II. - Eine Polynomfolge $\Phi_{1}(x), \ldots, \Phi_{n}(x), \ldots$ soll in einem Kreise um den Punkt $x=$ o gleichmässig gegen eine nicht identisch verschwindende Grenzfunktion konvergieren, und die zugehörige Arcusmenge soll in einem der Gebiete $A, B, C$, enthalten sein.

$$
\text { Gebiet } A \text { beisst das Intervall }(-\Theta,+\Theta) \text {, wo } \Theta<\frac{\pi}{2} \text {. }
$$

4) G. VItali, Sopra le serie di funzioni analitiche [Annali di Matematica pura ed applicata, Series III, Bd. X (1904), S. 65-82]. - Vgl. ferner: C. Carathéodory und E. Landau, Beiträge zur Konvergenz von Funktionenfolgen [Sitzungsberichte der Kgl. Preussischen Akademie der Wissenschaften (Berlin), Jahrgang igir, S. 587-613] und die Bemerkung von Carathéodory, loc. cit. ${ }^{x}$ ), b, Satz IV.

5) Insofern nicht das Gegenteil bemerkt wird, brauchen die Polynome $\Phi_{n}(x)$ jetzt nicht mehr die spezielle Gestalt ( $\mathrm{l}$ ) zu haben, wo $\Phi_{n}(0)=\mathrm{I}$ war. 
Gebiet $B$ ist das Intervall $\left(-\frac{\pi}{2},+\frac{\pi}{2}\right)$.

Gebiet $C$ besteht aus den $r$ getrennten Intervallen

$$
\begin{aligned}
\left(-\frac{\pi}{2 r}, \frac{\pi}{2 r}\right), \quad & \left(\frac{3 \pi}{2 r}, \frac{5 \pi}{2 r}\right), \ldots\left(\frac{(4 t-\mathrm{I}) \pi}{2 r}, \frac{(4 t+\mathrm{I}) \pi}{2 r}\right), \cdots \\
& \left(\frac{(4 r-5) \pi}{2 r}, \frac{(4 r-3) \pi}{2 r}\right) .
\end{aligned}
$$

(Es werden immer abgeschlossene Intervalle betrachtet).

Dann konvergiert die Polynomfolge $\Phi_{1}(x), \Phi_{2}(x), \ldots, \Phi_{n}(x), \ldots$ in der ganzen Ebene, gleichmässig in jedem endlichen Gebiet, und stellt eine ganze Funktion von endlichem Gescblecht dar. Das Geschlecht ist böchstens I, bzw. 2, 2r, und es konvergiert absolut die Summe der I-ten, bzw. 2-ten, 2 r-ten Potenzen der reziproken Nullstellen, je nacbdem die Arcusmenge in das Gebiet $A$, bzw. $B, C$ fällt.

Um Satz II zu beweisen, genügt es, die Voraussetzungen des Satzes I der Reihe nach in den drei Fällen zu verifizieren.

Im Falle $A$ setze ich voraus (das kann ich ohne Beschränkung der Allgemeinheit tun) dass die Folge $\Phi_{n}(x)$ in die Form (I) gesetzt ist, und man sieht dann unmittelbar, dass ${ }^{6}$ )

$$
\frac{\mathrm{I}}{\left|\alpha_{n 1}\right|}+\frac{\mathrm{I}}{\left|x_{n 2}\right|}+\cdots+\frac{\mathrm{I}}{\left|x_{n n}\right|} \leq \frac{\mathrm{I}}{\cos \theta}\left(\frac{\cos \beth_{n 1}}{\left|\alpha_{n 1}\right|}+\frac{\cos \Xi_{n 2}}{\left|\alpha_{n 2}\right|}+\cdots+\frac{\cos \beth_{n n}}{\left|x_{n n}\right|}\right)=\frac{\mathrm{I}}{\cos \theta} \Re\left(a_{n 1}\right) .
$$

Im Falle $B$ betrachte ich (von einem gewissen $n$ an) die Polynomfolge

$$
\begin{aligned}
\frac{\Phi_{n}(x-d)}{\Phi_{n}(-d)} & =\left(\mathrm{I}-\frac{x}{\alpha_{n 1}+d}\right)\left(\mathrm{I}-\frac{x}{\alpha_{2 n}+d}\right) \cdots\left(\mathrm{I}-\frac{x}{\alpha_{n n}+d}\right) \\
& =\mathrm{I}-b_{n 1} x+b_{n 2} x^{2}-\cdots+(-\mathrm{I})^{n} b_{n n} x^{n} .
\end{aligned}
$$

Ich kann für $d$ eine positive Zahl wählen, im Innern des Kreises, worin Konvergenz vorausgesetzt wurde, und derart, dass

Ich setze

$$
\lim _{n=\infty} \Phi_{n}(-d) \neq 0 .
$$

dann hat man

also

$$
x_{n p}+d=\xi_{n p}+i n_{n p}
$$

$$
\begin{gathered}
b_{n \mathrm{I}}=\sum_{p=1}^{n} \frac{\mathrm{I}}{\alpha_{n p}+d}=\sum_{p=1}^{n} \frac{\xi_{n p}-i n_{n p}}{\left|x_{n p}+d\right|^{2}}, \\
\left|b_{n 1}\right| \gg \sum_{p=1}^{n} \frac{\xi_{n p}}{\left|x_{n p}+d\right|^{2}} \gg d \sum_{p=1}^{n} \frac{I}{\left|x_{n p}+d\right|^{2}}
\end{gathered}
$$

$$
\sum_{p=1}^{n} \frac{1}{\left|\alpha_{n p}+d\right|^{2}} \leq \frac{\left|b_{n 1}\right|}{d}
$$

6) Vgl. 1. c. ${ }^{\mathbf{I}}$ ), b), Satz I. 
So sind alle Voraussetzungen des Satzes I mit dem Exponenten $k=2$ für die Folge $\frac{\Phi_{n}(x-d)}{\Phi_{n}(-\bar{d})}$ verifiziert.

Liegen endlich die Wurzeln des Polynoms $\Phi_{n}(x)$ im Gebiet $C$, so setze ich $\omega=e^{\frac{2 \pi i}{r}}, z=x^{r}$, und dann liegen die Wurzeln des Polynoms der Variablen $z$

$$
\Phi_{n}(x) \Phi_{n}(\omega x) \Phi_{n}\left(\omega^{2} x\right) \ldots \Phi_{n}\left(\omega^{r-1} x\right)=c_{n 0}-c_{n 1} z+\cdots+(-\mathrm{I})^{n} c_{n n} z^{n}
$$

d. h. die Zahlen

$$
\alpha_{n 1}^{r}, \quad \alpha_{n 2}^{r}, \ldots, \alpha_{n n}^{r}
$$

in dem Gebiete $B$. Daraus folgt, dass es eine positive Zahl $d$ gibt, sodass für alle $n$

$$
\frac{1}{\left|x_{n 1}^{r}+d\right|^{2}}+\frac{1}{\left|x_{n 2}^{r}+d\right|^{2}}+\cdots+\frac{1}{\left|x_{n n}^{r}+d\right|^{2}}<\text { Konst. }
$$

Nun unterscheide ich zwei Fälle. Verschwindet die Grenzfunktion im Punkte $x=0$ nicht, so ist für alle hinreichend grosse $n$

also

$$
\left|x_{n p}\right|>p
$$

$$
\sum_{p=1}^{n} \frac{I}{\left|\alpha_{n p}\right|^{2 r}} \leq \sum_{p=1}^{n} \frac{I}{\left|\alpha_{n p}\right|^{2 r}} \frac{\left(I+\frac{d}{\rho^{r}}\right)^{2}}{\left|I+\frac{d}{\alpha_{n p}^{r}}\right|^{2}} \leq\left(\mathrm{I}+\frac{d}{\rho^{r}}\right)^{2} \text {. Konst. }
$$

Wenn aber der Punkt o eine $m$-fache Wurzel der Grenzfunktion ist, so kann man von $\Phi_{n}(x)$ einen gegen $x^{m}$ konvergierenden Faktor abspalten, und die Voraussetzung des Satzes I mit $k=2 r$ für das zurückbleibende Polynom verifizieren.

Das Resultat für das Gebiet $C$ hat uns Herr Fekete freundlichst mitgeteilt ${ }^{7}$ ).

Zusatz: Es sollen dieselben Bedingungen betreffend die Konvergenz und die Grenzfunktion der Folge $\Phi_{1}(x), \ldots, \boldsymbol{\Phi}_{n}(x), \ldots$ bestehen, als im Satz II. Ueber die Lage der Wurzeln soll aber jetzt die allgemeinere Voraussetzung gemacbt werden, dass die Arcusse von böchstens $T$ Wurzeln des annäbernden Polynoms $\Phi_{n}(x)$ aus dem Gebiete $A$ (bzw. $B, C$ ) binaustreten können $(n=1,2,3, \ldots)$ - Dann besteht noch immer die im Satz II angegebene Konsequenz.

Um dies z. B. für das Gebiet $A$ zu beweisen, nehme ich $\Phi_{n}(x)$ in der Form an $\Phi_{n}(x)=\left(\mathrm{I}-\frac{x}{\alpha_{n \mathrm{t}}}\right) \ldots\left(\mathrm{I}-\frac{x}{\alpha_{n n}}\right)\left(\mathrm{I}-\frac{x}{\beta_{n 1}}\right) \ldots\left(\mathrm{I}-\frac{x}{\beta_{n T}}\right)=\mathrm{I}-a_{n 1} x+\cdots$ wo die Wurzeln $\alpha_{n 1}, \ldots, \alpha_{n n}$ ihre Arcusse zwischen $-\theta$ und $+\Theta$ haben $\left(\theta<\frac{\pi}{2}\right)$, während über die Lage von $\beta_{n 1}, \ldots, \beta_{n T}$ nichts vorausgesetzt wird. Jedoch müssen (wegen der Voraussetzung über die gleichmässige Konvergenz) von

7) Er fand es in Kenntniss aller unserer vorhergehenden Resultate. Vgl. Hauptsàchlich die Bemerkung über die Abschnitte von Potenzreihen in der Arbeit loc. cit. a), am Ende von 6. 
einem gewissen $n$ an die Ungleichungen bestehen

Nun ist

$$
\left|\beta_{n q}\right|>p>0 \quad(q=1,2, \ldots, T) .
$$

$$
\frac{\Re\left(\sum_{p=1}^{n} \frac{\mathrm{I}}{\boldsymbol{x}_{n p}}\right)}{\cos \theta}=\frac{\Re\left(a_{n 1}-\sum_{q=1}^{T} \frac{\mathrm{I}}{\beta_{n q}}\right)}{\cos \Theta} \geqslant \frac{\mathrm{I}}{\left|\mathbf{x}_{n 1}\right|}+\frac{\mathrm{I}}{\left|\mathrm{x}_{n 2}\right|}+\cdots+\frac{\mathrm{I}}{\left|\mathbf{x}_{n n}\right|}
$$

und folglich

$$
\frac{\left|a_{n 1}\right|+\frac{T}{p}}{\cos \Theta}+\frac{T}{p} \geqslant \frac{\mathrm{I}}{\left|a_{n 1}\right|}+\cdots+\frac{\mathrm{I}}{\left|\alpha_{n n}\right|}+\frac{\mathrm{I}}{\left|\beta_{n 1}\right|}+\cdots+\frac{\mathrm{I}}{\left|E_{n T}\right|} .
$$

Damit ist auch dieser Zusatz zum Satz II bewiesen. Satz II sagt also (unter anderem) aus, dass wenn die Polynome einer Folge alle ihre Wurzeln (höchstens mit einer gleichmässig beschränkten Anzahl von Ausnahmen) in einem Winkelraum mit der

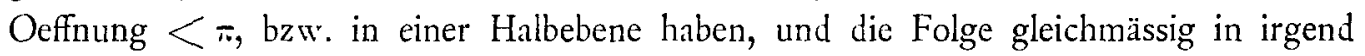
einem Gebiete ausserbalb des Winkelraumes bzw. der Halbebene konvergiert und die Grenzfunktion nicht identisch verschwindet ${ }^{8}$ ), so konvergiert dieselbe Folge notwendigerweise in der ganzen Ebene, und die Grenzfunktion ist höchstens vom Geschlecht I bzw. 2. Daraus folgt ohne Weiteres der

SATz III. - Konvergiert eine Folge von Polynomen, deren keines mebr imaginäre (d.b. nicht reelle) Wurzeln hat als $T$, in irgend einem Gebiete der Ebene gleichmässig gegen eine nicht identisch verschuindende Grenzunktion, so konvergiert sie in der ganzen Ebene. Die Grenzfunktion ist gleich dem Produkte einer ganzen Funktion vom Geschlecht $\leq \mathrm{I}$, und der Funktion $e^{-\Upsilon x^{2}}$, wo $\gamma \geqslant 0^{9}$ ).

Dies ist auf Grund des Vorangegangenen evident - bis auf die Behauptung, dass

8) Man halte nur immer die Folge $x, x^{2}, \ldots, x^{n}, \ldots$ vor Augen. Für ganze Funktionen vom Geschlecht I gilt die Aussage ebenfalls nicht mehr. Beispiel :

$$
\Phi_{n}(x)=\mathrm{I}-e^{n i x}
$$

Sämtliche Wurzeln von $\Phi_{n}(x)$ liegen auf der Geraden $\eta=0 ; \lim _{n=\infty} \Phi_{n}(x)=\mathrm{I}$, wenn $\eta>0, \lim _{n=\infty} \Phi_{n}(x)$ nicht vorhanden, wenn $\eta \leqslant 0\left(x=\xi+i r_{1}\right)$.

9) E. Laguerre, Euvres (Paris, Gauthier-Villars), Bd. I (1898), S. I74-177; G. Pólya, loc. cit. '), a), Satz II. - In letzterer Arbeit wurde die Frage aufgeworfen ob der Satz der vorliegenden Arbeit besteht oder nicht; vgl. Anfang von 7. Für reelle Wurzeln eines Zeichens wurde die Frage gelöst durch den Satz II in Arbeit loc. cit. ' $)$, b). - Man vgl. noch M. Ретrovitch, Une classe remarquable de séries entières [Atti del IV Congresso dei Matematici (Roma, 6-I I aprile 1908), Bd. II (1909), S. 36-43] und a, Sātze III und IV. Bezüglich der zuletzt angeführten Untersuchungen bemerken wir: aus dem Sātze III der vorliegenden Arbeit folgt die Alternative: wenn eine Potenzreihe unendlich viele Abschnitte mit nur reellen Wurzeln hat, dann ist ihr Konvergenzradius $\rho$ entweder o oder $\infty$. Man schliesst die erste Möglichkeit $(\rho=0)$ aus und gewinnt viel allgemeinere ăhnliche Sătze auf Grund der Bemerkung: " Sind unendlich viele Abschnitte einer Potenzreihe für $|x|=R$ unter einer festen Grenze gelegen, so konvergiert die Potenzreihe für $|x|<R »$. 
$\gamma \geqslant 0$ ist. Der einfache Beweis dieser Behauptung für $T=0$ ist aber schon an anderer Stelle erbracht ${ }^{10}$ ).

Satz IV. - Ist eine Polynomfolge gleichmässig konvergent in einem Gebiete, das den Punkt $x=0$ im Innern enthält, aber nicht in der gañen Ebene konvergent, so muss die zweite derivierte Menge der zugebörigen Arcusmenge noch Punkte enthalten "I). (Dabei wird wieder vorausgesetzt, dass die Grenzfunktion nicht identisch verschwindet).

Denn sollte dies nicht der Fall sein, so hätte die Arcusmenge nur eine endliche Anzahl von Häufungspunkten, $\Xi_{1}, \Xi_{2}, \ldots, \Xi_{l}$. Man könnte dann $l+\mathrm{I}$ ganze Zahlen ${ }^{\mathrm{I}}$ )

$$
q, p_{1}, p_{2}, \ldots, p_{l} \quad(q>0)
$$

so bestimmen, dass die Ungleichungen

oder auch die Ungleichungen

$$
\left|q \frac{\Phi_{1}}{2 \pi}-p_{1}\right| \leq \frac{\mathrm{I}}{q^{\frac{l}{l}}}, \ldots,\left|q \frac{\Phi_{l}}{2 \pi}-p_{l}\right| \leq \frac{\mathrm{I}}{q^{\frac{\mathrm{I}}{l}}}
$$

$$
\begin{gathered}
\left|q \frac{\Phi_{1}}{2 \pi}-p_{1}\right|<\frac{\mathrm{I}}{4}, \ldots,\left|q \frac{\Xi_{l}}{2 \pi}-p_{l}\right|<\frac{\mathrm{I}}{4}, \\
\frac{\left(4 p_{\mathrm{I}}-\mathrm{I}\right) \pi}{2 q}<{\Xi_{\mathrm{I}}}_{\mathrm{r}}<\frac{\left(4 p_{1}+\mathrm{I}\right) \pi}{2 q}, \ldots, \frac{\left(4 p_{l}-\mathrm{I}\right) \pi}{2 q}<\Im_{l}<\frac{\left(4 p_{l}+\mathrm{I}\right) \pi}{2 q}
\end{gathered}
$$

bestehen. Dies besagt aber, dass von einem gewissen $n$ an die ganze Arcusmenge in einem Gebiet $C$ liegt. Dann würde aber die Polynomfolge in der ganzen Ebene konvergieren, im Widerspruche zu unserer Voraussetzung.

Bei speziellen Polynomfolgen erlaubt aber der Satz II noch viel schärfere Konsequenzen zu ziehen. Es besteht z. B. der

Satz V. - Die Potenzreibe

$$
a_{0}+a_{1} x+a_{2} x^{2}+\cdots+a_{n} x^{n}+\cdots
$$

habe positive nie abnebmende Koeffizienten, und sei im Kreise $|x|<$ I konvergent. Man bilde die Menge $M$ sämtlicher Wurzeln sämtlicher Abschnitte; die derivierte Menge $M^{\prime}$ dieser Menge besteht aus den Wurzeln der Potenzreibe im Innern des Einheitskreises und aus dem ganzen Rande des Einbeitskreises ${ }^{\mathbf{1 3}}$ ).

Nach einem algebraischen Satz ${ }^{\mathbf{1 4}}$ ) wird die Menge $M$ gänzlich im Einheitskreis enthalten sein. Wir haben nur zu beweisen, dass jeder Punkt des Kreisrandes zur Menge $M^{\prime}$ gehört. Sollte aber dies nicht der Fall sein, so würde ein ganzer Bogen existieren, der nicht zu $M^{\prime}$ gehört; man könnte nun durch die beiden Endpunkte

Io) Vgl. loc. cit. ' $)$, a) den Beweis für Satz I und das Ende des Beweises für Satz II.

I I) Die unendlich oft vorkommende Arcusse werden zu der ersten derivierten Menge gerechnet.

12) H. Mrnkowski, Diophantische Approximationen (Leipzig, Teubner, I907), S. 8.

r3) Vgl. loc. cit. I), b), Satz III.

14) S. Kakeya, On the Limits of the Roots of an Algebraic Equation with Positive Coefficients [The Tóhoku Mathematical Journal, Bd. II (I9r2-1913), S. 140-142]. 
eines kleineren Bogens eine Gerade ziehen, die die Ebene in zwei Teile teilt, derart, dass sämtliche Abschnittswurzeln von einem gewissen $n$ an in die eine Halbebene fallen, während die andere Halbebene ein Stück des Konvergenzkreises enthält. Daraus würde aber folgen (nach Satz II), dass die Potenzreihe überall konvergiert, was gewiss nicht der Fall ist.

Beispiele zu Satz V bieten die geometrische Reihe und alle ihre Derivierten dar.

Göttingen, den 24. Juli 1913 .
E. LINDWART.
G. PólY A. 